\title{
Progress towards a semiconductor Compton camera for prompt gamma imaging during proton beam therapy for range and dose verification
}

\author{
A. Gutierrez, ${ }^{a 1}$ C. Baker, ${ }^{b}$ H. Boston, ${ }^{c}$ S. Chung, ${ }^{a}$ D.S. Judson ${ }^{c}$, A. Kacperek, ${ }^{d}$ \\ B. Le Crom, ${ }^{c}$ R. Moss, ${ }^{a}$ G. Royle, ${ }^{\text {a }}$ R. Speller, ${ }^{a}$ A. J. Bostonc \\ ${ }^{a}$ Department of Medical Physics and Biomedical Engineering, University College London \\ London, WC1E 6BT, United Kingdom \\ ${ }^{b}$ Medical Physics Department, Royal Berkshire NHS Foundation Trust \\ Reading RG1 5AN, United Kingdom \\ ${ }^{c}$ Department of Physics, University of Liverpool \\ Liverpool, L69 3BX, United Kingdom \\ ${ }^{d}$ The National Eye Proton Therapy Centre, Clatterbridge Cancer Centre NHS Foundation Trust \\ Wirral, CH63 4JY, United Kingdom \\ E-mail: a.gutierrez@ucl.ac.uk
}

\begin{abstract}
The main objective of this work is to test a new semiconductor Compton camera for prompt gamma imaging. Our device is composed of three active layers: a $\mathrm{Si}(\mathrm{Li})$ detector as a scatterer and two high purity Germanium detectors as absorbers of high-energy gamma rays. We performed Monte Carlo simulations using the Geant 4 toolkit to characterise the expected gamma field during proton beam therapy and have made experimental measurements of the gamma spectrum with a $60 \mathrm{MeV}$ passive scattering beam irradiating a phantom. In this proceeding, we describe the status of the Compton camera and present the first preliminary measurements with radioactive sources and their corresponding reconstructed images.
\end{abstract}

KEYWORDS: Compton camera; semiconductor detector; prompt gamma; Monte Carlo; Geant4;

\footnotetext{
${ }^{1}$ Corresponding author.
} 


\section{Contents}

1. Introduction 1

2. Gamma emission during proton beam therapy 1

2.1 Simulations of gamma spectrum 2

2.2 Experimental measurements of gamma spectrum 2

2.3 Spatial distribution of gamma emission 4

3. Semiconductor Compton camera 4

3.1 Compton camera principle of operation for 3D imaging 4

3.2 ProSPECTus-based Compton camera for prompt gamma imaging 5

3.3 Image reconstruction using Stochastic Origin Ensemble $\quad 6$

$\begin{array}{lr}\text { 4. Conclusion } & 8\end{array}$

\section{Introduction}

Proton beam therapy (PBT) has considerable clinical benefits over photon therapy due to the localised nature of energy deposition in the Bragg peak. This spares surrounding healthy tissues and critical organs unwanted radiation. However, the full potential of PBT is currently limited by uncertainties associated with dose deposition which can result in erratic dose delivery in the tumor and/or healthy tissue [1]. In-vivo real-time knowledge of proton dose deposition, which would enable online correction and personalisation of treatment is highly desirable [2].

During proton irradiation, characteristic prompt gamma rays are produced due to deexcitation of nuclei in the tissue. The spatial distribution of prompt gamma rays is highly correlated to the proton's range and can potentially be used for verification of the proton dose [3].

Several detectors are under development to detect prompt gamma rays, including knife edge gamma cameras [4], multi-slit collimator gamma cameras [5] and double and triple stage Compton cameras [6][7]. The challenge remains in optimising the device for detecting prompt gamma rays, the energies of which range from 1 to $10 \mathrm{MeV}$. Recently, a knife edge gamma camera was successfully tested during PBT, demonstrating the potential of using prompt gamma rays as a verification tool [4].

\section{Gamma emission during proton beam therapy}

Monte Carlo simulations using the Geant4 toolkit [8] were performed to characterise the prompt gamma rays produced during proton irradiation. Geant4 version 10.02.p02 and physics list QGSP_BERT_HP_LIV were used. We simulated paraffin and water phantoms irradiated with $60 \mathrm{MeV}$ and $150 \mathrm{MeV}$ protons and tracked the gamma rays leaving the phantom. In the simulations, we concentrate on the production of gamma rays inside the target and beam line models have not been included, which neglects any gamma rays and neutrons that are generated 
in the beam line. Experimental measurements of the gamma spectrum were performed at the National Eye Proton Therapy Centre in the Clatterbridge Cancer Centre [9], where a paraffin and a water target were irradiated with $60 \mathrm{MeV}$ protons.

\subsection{Simulations of gamma spectrum}

After nuclear inelastic interactions, prompt gamma rays are emitted during the deexcitation of nuclei in the tissue. Prompt gamma rays have characteristic energies corresponding to nuclear levels of atoms present in tissue. For example, a ${ }^{12} \mathrm{C}$ target produces primarily 4.44 MeV and 2.00 MeV gamma rays, while a ${ }^{16} \mathrm{O}$ target produces mostly $6.13 \mathrm{MeV}, 6.92 \mathrm{MeV}$, 7.12 MeV, $2.74 \mathrm{MeV}$ and $4.44 \mathrm{MeV}$ gamma rays [10]. Gamma rays are also produced in other interactions such as positron annihilation, neutron capture, activation and Bremsstrahlung.

Figure 1 shows the simulated energy spectra of gamma rays leaving $(10 \mathrm{~cm} \times 10 \mathrm{~cm} \times 20 \mathrm{~cm})$ water $\left(1.00 \mathrm{~g} / \mathrm{cm}^{3}, \mathrm{H}_{2} \mathrm{O}\right)$ and paraffin $\left(0.93 \mathrm{~g} / \mathrm{cm}^{3}, \mathrm{C}_{\mathrm{n}} \mathrm{H}_{2 \mathrm{n}+2}\right)$ phantoms. The phantoms were independently irradiated at two different proton energies, $60 \mathrm{MeV}$ and $150 \mathrm{MeV}$. As expected, the composition and density of the material have a strong influence on the prompt gamma yield. There are no gamma lines from ${ }^{16} \mathrm{O}$ due to the absence of this atom in paraffin. In contrast, the 4.44 MeV peak is more pronounced in paraffin than water since the $4.44 \mathrm{MeV}$ peak in water is due to an ${ }^{16} \mathrm{O}$ transformed into an excited ${ }^{12} \mathrm{C}$, while ${ }^{12} \mathrm{C}$ is already present in paraffin. We can also see that the incident energy of protons plays a key role in the prompt gamma yield, as the gamma lines are more prominent at $150 \mathrm{MeV}$ than $60 \mathrm{MeV}$, as well as the $2.2 \mathrm{MeV}$ line, corresponding to neutron capture in hydrogen.Figure 1
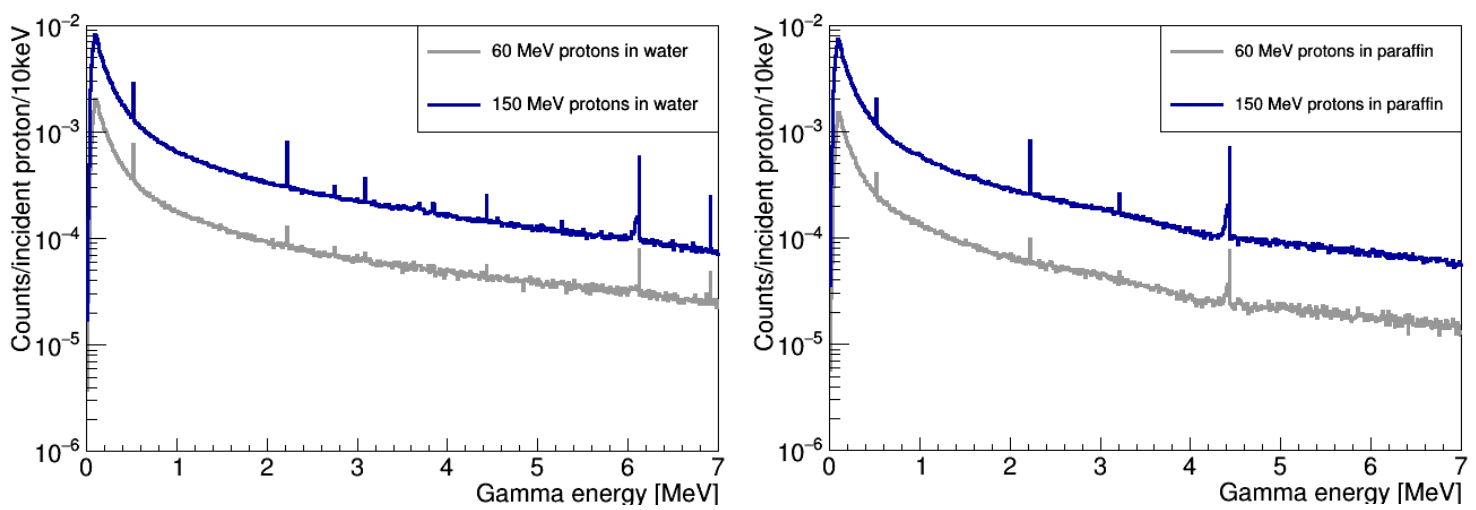

Figure 1. Geant4 simulations of the energy of gamma rays leaving a water phantom (left) and a paraffin (right) phantom.

\subsection{Experimental measurements of gamma spectrum}

An experimental gamma spectrum was obtained by irradiating a cylindrical paraffin target $(10 \mathrm{~cm}$ diameter and $19 \mathrm{~cm}$ height, see Figure $2 \mathrm{~b})$ and a water target $(16 \mathrm{~cm}$ diameter and $19 \mathrm{~cm}$ height, see Figure 2c) with $60 \mathrm{MeV}$ protons at the Clatterbridge Cancer Centre. A coaxial HPGe spectrometer from ORTEC (trans-SPEC-DX-100T), which is $6.5 \mathrm{~cm}$ in diameter and $5 \mathrm{~cm}$ thick, was used for gamma detection. The Clatterbridge medical beam line is a passive scattering beam providing protons up to $60 \mathrm{MeV}$ to treat eye tumors. We used a $1.5 \mathrm{~cm}$ diameter collimator and the paraffin/water target was placed $20 \mathrm{~cm}$ from the nozzle (see Figure 2a). The HPGe detector was placed at $50^{\circ}$ to the beam line, $43 \mathrm{~cm}$ from the target, as illustrated in Figure 2d. 


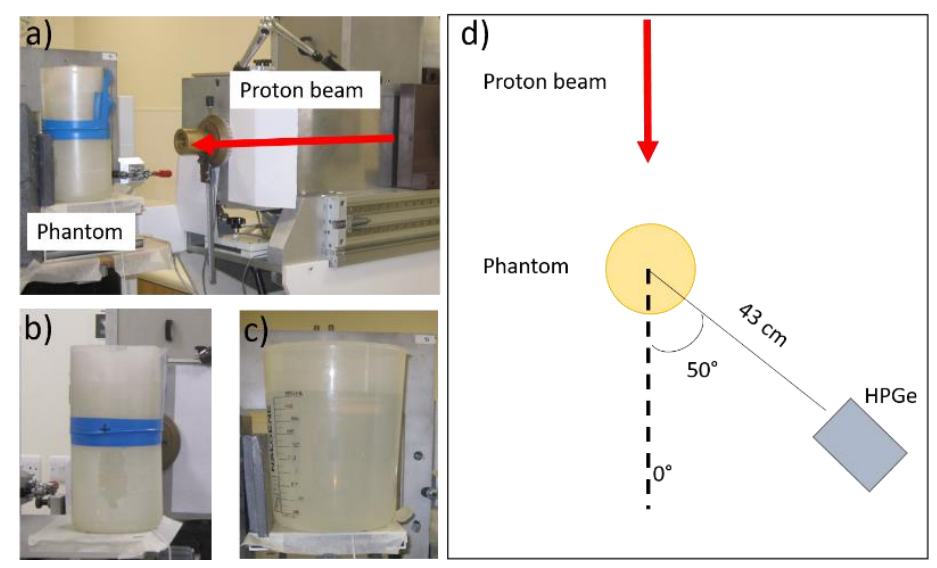

Figure 2. a) Experimental set up at Clatterbridge Cancer Centre. b) Paraffin phantom and c) water phantom. d) Illustration of the top view of the experimental set up (not to scale).

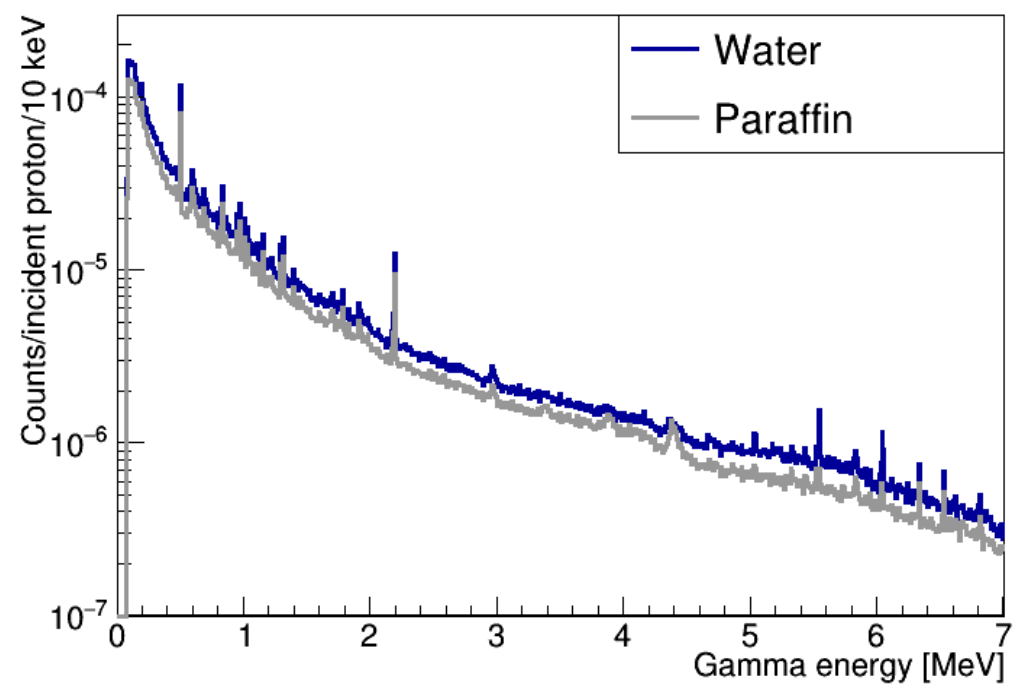

Figure 3. Energy spectrum of gamma rays detected by the HPGe trans-SPEC-DX-100T detector when a water and paraffin target are irradiated by $60 \mathrm{MeV}$ protons at the Clatterbridge Cancer Centre.

The phantoms were independently irradiated with about $1 \times 10^{9}$ protons and the resulting gamma ray spectrum is shown in Figure 3. Although we cannot make a direct comparison with the simulations (Figure 1), we can still make some qualitative remarks. We can see that for both spectra, the $511 \mathrm{keV}$ and $2.2 \mathrm{MeV}$ gamma lines are very visible and there are several lines between $511 \mathrm{keV}$ and $2 \mathrm{MeV}$ that are not present in the simulation. Also, the $4.4 \mathrm{MeV}$ peak is broader and more important in paraffin than water because of the composition of the target. The gamma continuum in the background seems to follow the same trend as the simulations, with a Bremsstrahlung peak at around $100 \mathrm{keV}$. The differences between the simulated spectrum and the experimental data can potentially be explained by the fact that the beam line model was not included in the simulation and therefore have not accounted for any gamma rays and neutrons generated in the beam line, or any gamma rays from activation of the phantom material following irradiation [11]. Other gamma peaks detected can be associated to the beam line materials such as the $0.84 \mathrm{MeV}$ and $1.01 \mathrm{MeV}$ from ${ }^{27} \mathrm{Al}$ and, $0.67 \mathrm{MeV}$ and $0.99 \mathrm{MeV}$ from ${ }^{63} \mathrm{Cu}$ and ${ }^{64} \mathrm{Zn}$, 
respectively. Also, it is known prompt gamma cross sections used in Geant4 need further validation [12][13].

\subsection{Spatial distribution of gamma emission}

Prompt gamma rays are correlated to the proton range and dose but do not directly reflect energy deposition: prompt gamma rays are generated in nuclear interactions while the proton's Bragg peak is due to Coulomb interactions with atomic electrons. Figure 4 shows the gamma ray emission and the relative dose deposited by $60 \mathrm{MeV}$ protons in water. The falloff in gamma ray emission corresponds to the energy threshold for the nuclear interactions. As protons undergo lateral spread near the end of their range, lateral spread on the gamma ray yield is also observed, as shown in Figure 4.
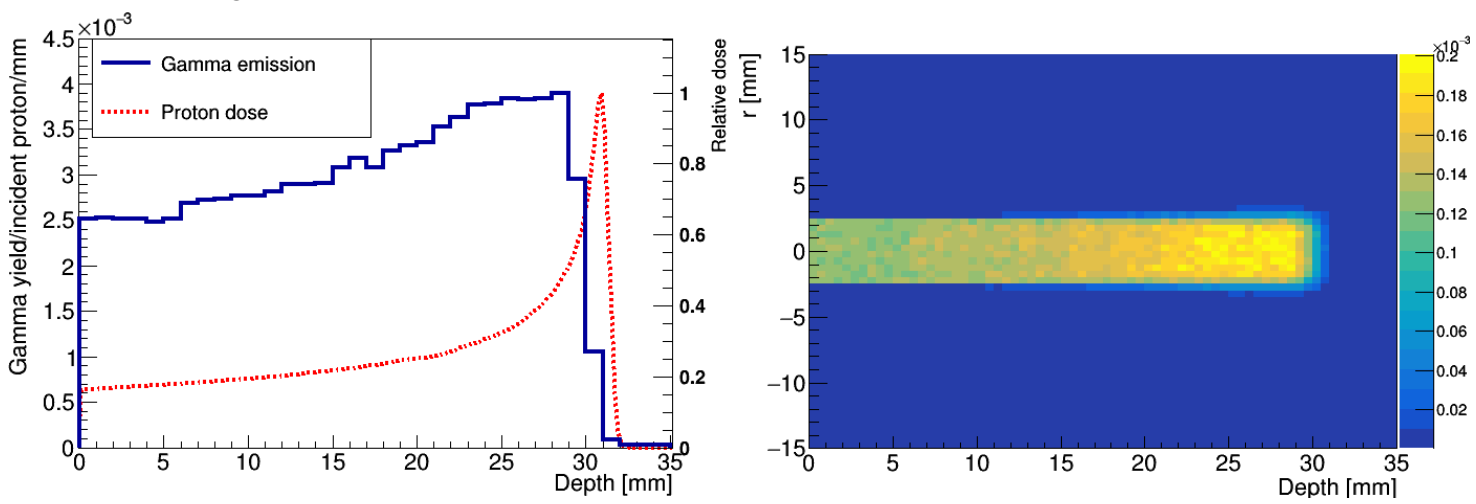

Figure 4. Geant4 simulations of the gamma yield and proton relative dose as a function of depth (left) and radial profile of gamma yield as a function depth using a $5 \mathrm{~mm}$ wide beam (right).

Clearly here is a need to develop techniques that allow the proton dose to be estimated from observations of the gamma ray distribution, otherwise gamma ray imaging remains a technique to estimate a relative change of proton range rather than the absolute range. The remainder of this paper looks at the reconstruction of gamma distributions with a Compton camera.

\section{Semiconductor Compton camera}

\subsection{Compton camera principle of operation for 3D imaging}

Compton cameras exploit Compton kinematics to track back the location of the gamma source. The device is generally composed of two stages, a scatterer and an absorber. In the scatterer, a recoil electron, with energy $e_{1}$, is detected after the initial photon is scattered by an angle $\theta$. The scattered photon, with energy $e_{2}$, is then fully detected at the second stage by the absorber. The concept of a Compton camera is shown in Figure 5. Both detectors are position and energy sensitive so the direction and angle $\theta$ of the scattered photon can be calculated as

$$
\cos \theta=1-m_{e} c^{2}\left[\frac{1}{e_{2}}-\frac{1}{e_{1}+e_{2}}\right],
$$

where $m_{e}$ is the electron rest mass, $c$ is the speed of light, $e_{2}$ is the energy of the scattered gamma $E_{\gamma}$, and $e_{1}+e_{2}$ is the energy of the incident gamma ray $E_{\gamma}$. This information is then used to reconstruct a conical surface representing possible positions where the gamma ray was emitted. Multiple events will lead to an overlap of conical surfaces, with a higher density at the actual source position. There are different techniques to reconstruct images from Compton cameras, such as simple back projection of conical surfaces or iterative algorithms using statistical methods 
(e.g. maximum likelihood expectation maximisation and stochastic origin ensemble). A review of different reconstruction algorithms can be found in [14]. For this work, we use the stochastic origin ensemble (SOE), since it has been demonstrated that it can improve the spatial resolution using a fast algorithm, which is a significant advantage for near-real-time image reconstruction during treatment [15].

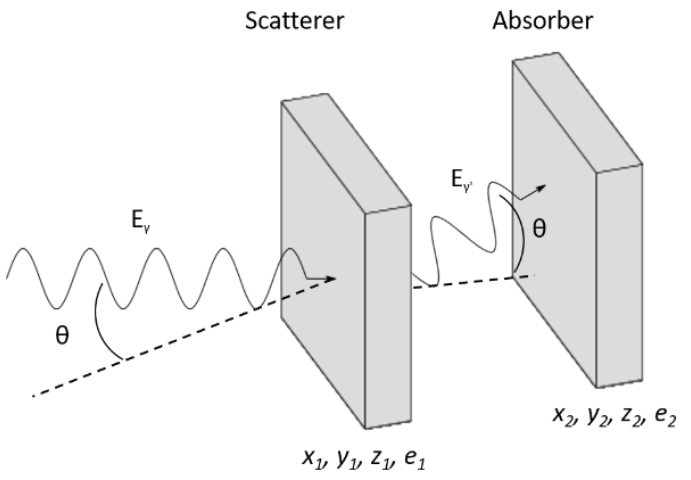

Figure 5. Illustration of a Compton camera. Both detectors are position $(x, y, z)$ and energy sensitive $(e)$.

\subsection{ProSPECTus-based Compton camera for prompt gamma imaging}

ProSPECTus is a Compton camera developed and optimised for Single Photon Emission Computed Tomography (SPECT) of radioisotopes, such as ${ }^{99 \mathrm{~m}} \mathrm{Tc}$, which emits $141 \mathrm{keV}$ gamma rays [16]. In this work, we used the same camera with an additional absorber to fully absorb the prompt gamma rays. The device is composed of three stages:

1) The scatterer is a double sided strip Silicon-Lithium ( $\mathrm{Si}(\mathrm{Li})$ ) detector from CANBERRA. Its active area and thickness are $3500 \mathrm{~mm}^{2}$ and $8 \mathrm{~mm}$ respectively, with a diameter of $71 \mathrm{~mm}$. Each face is segmented in 13, with a strip pitch of $5 \mathrm{~mm}$, separated by $500 \mu \mathrm{m}$. The detector is encased in an aluminium cryostat and is cooled by CANBERRA's CryoPulse 5 (CP5) electrical cooler which is connected to one side of the detector.

2) The first absorber is a double sided strip High-Purity Germanium (HPGe) detector from CANBERRA. The detector is $20 \mathrm{~mm}$ thick with an active area of $(60 \times 60) \mathrm{mm}^{2}$. Each face is segmented in 12 strips and each strip pitch is $5 \mathrm{~mm}$ separated by $500 \mu \mathrm{m}$. The detector is encased in an aluminium cryostat and is cooled by the CP5 connected to one side of the detector.

3) The second absorber is a coaxial HPGe crystal with a diameter of $66.5 \mathrm{~mm}$ and $50 \mathrm{~mm}$ thick, also provided by CANBERRA and cooled by the CP5. The detector does not have position resolution so it is used to absorb the photons scattered in the first absorber. The energy $e_{2}$ required for image reconstruction is the sum of the energies deposited in both absorbers.

The data from the three detectors is acquired by CAEN V1724 digitisers and the coincidence signal is processed through CAEN V1495 VME board. The detector assembly is shown in Figure 6. 


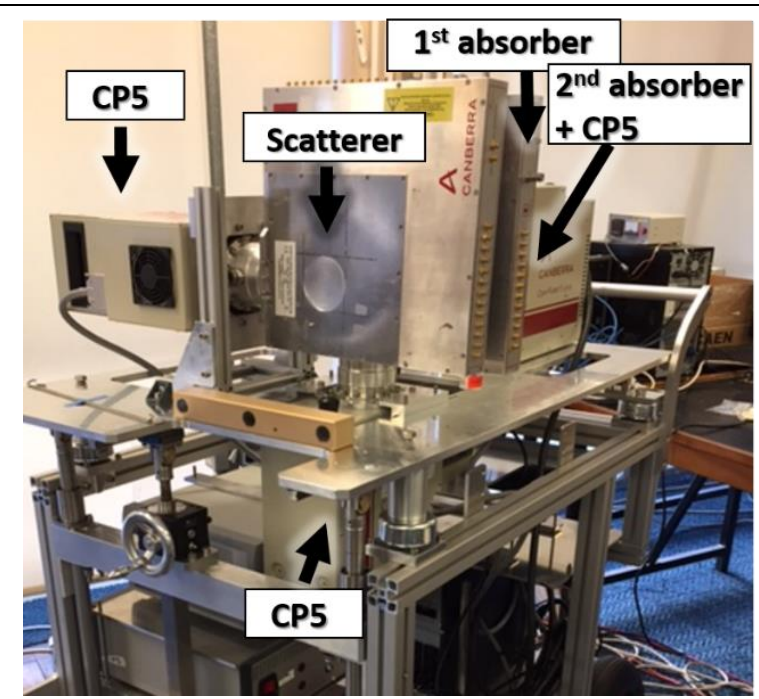

Figure 6. ProSPECTus-based Compton camera for high energy gamma rays.

\subsection{Image reconstruction using Stochastic Origin Ensemble}

We used the Stochastic Origin Ensemble (SOE) iterative algorithm to reconstruct images of gamma radioactive sources. SOE was developed for a Compton camera system by Andrvevy et al. and it is extensively described in [15]. We implemented the algorithm in C++ compiled software and ran it using one core of a $2.90 \mathrm{GHz}$ Intel i7-6920HQ Processor. The code first calculates the conical surface of each event and then randomly selects a point in that given surface. Since the surfaces overlap, a first random selection of points (one per event) will give a rough estimate of the source position. An iteration consists of selecting a new random point per event and by using the previous image density, the new point can be accepted or rejected. The new point will be always accepted if it migrates to a higher density position.

We performed two experimental measurements: First, a ${ }^{88} \mathrm{Y}$ radioactive source was placed $157 \mathrm{~mm}$ from the back of the absorber and about 112,000 events were recorded. Secondly, a ${ }^{57} \mathrm{Co}$ source and a ${ }^{139} \mathrm{Ce}$ source were placed $60 \mathrm{~mm}$ apart, $150 \mathrm{~mm}$ from the back of the absorber and around 92,000 events were recorded.

We used the SOE initialisation to perform a simple back projection (SBP) check by selecting multiple random points per event normalised by the circumference of the cone section present in the field of view. There are about 600 points per event and the algorithm runs in less than $6 \mathrm{~s}$ for a given distance. This is a very quick way to verify the data in comparison to conventional and time consuming SBP where the conical sections are traced voxel by voxel. The reconstructed images using SBP are shown in Figure 7a and Figure 9a. Figure 7b and Figure 7c show the SOE image reconstruction of ${ }^{88} \mathrm{Y}$ source after its initialisation and after 50 iterations, with a processing time of $\sim 1 \mathrm{~s}$ and $\sim 2 \mathrm{~s}$, respectively (the processing time may vary depending on the number of events and the image resolution). Figure 8 shows a horizontal slice of each case, we can see that the Full Width Half Maximum (FWHM) decreases from $24.8 \pm 1.7 \mathrm{~mm}$ (0 iterations) to $15.6 \pm 2.9 \mathrm{~mm}$ (after 50 iterations), where the errors are the FWHM statistical fluctuations of SOE in 10 samples. The SOE algorithm also efficiently reduces the tails of the distributions. 


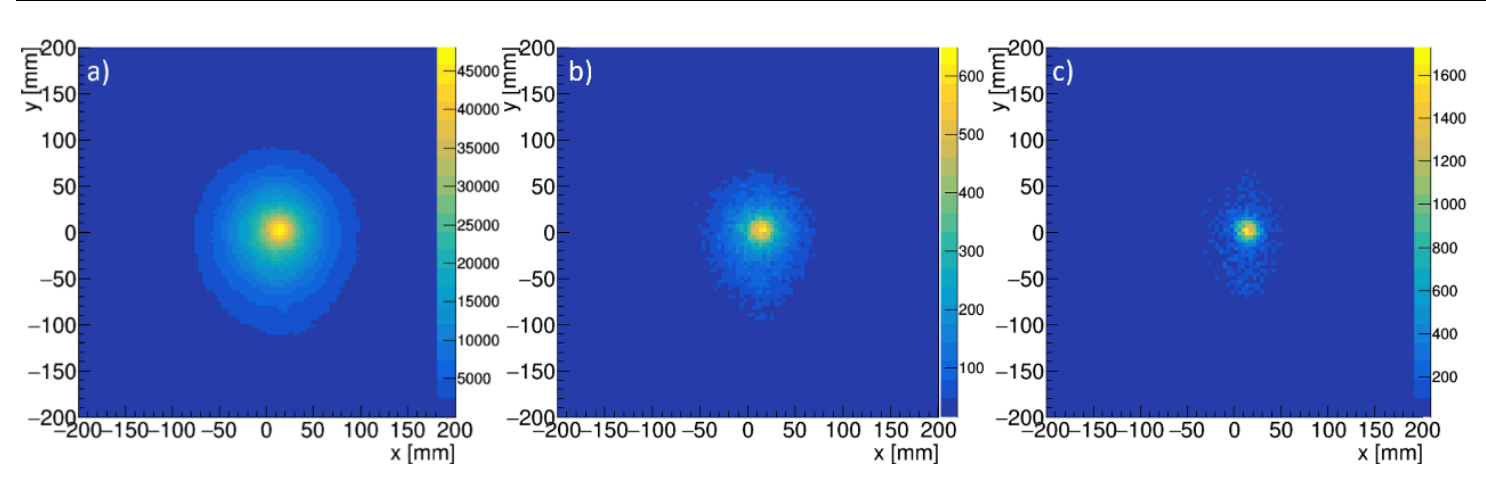

Figure 7. Image reconstruction of ${ }^{88} \mathrm{Y}$ source. a) is Simple back projection using SOE initialisation (multiple points per event), b) is the image from SOE initilisation and c) is the image after applying 50 iterations.

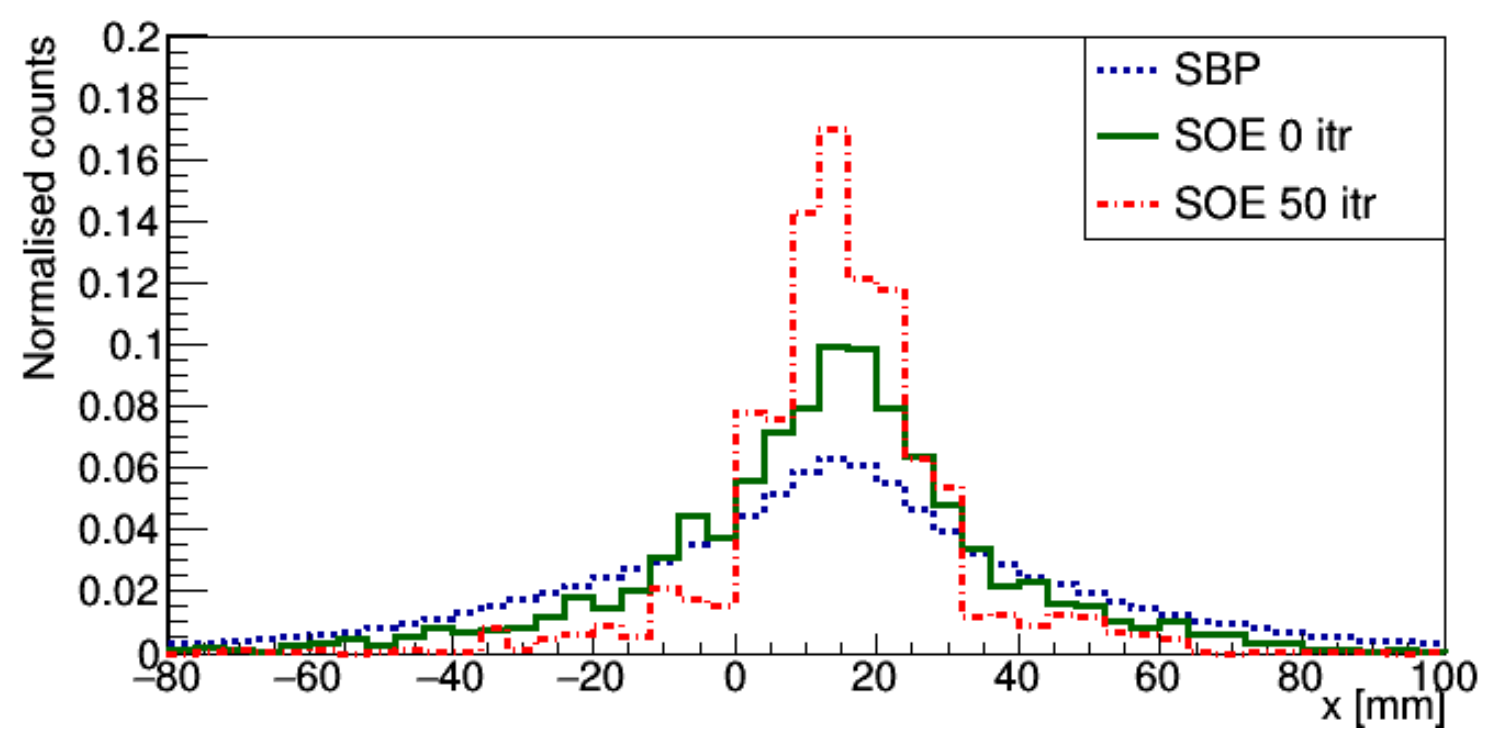

Figure 8. Profile histogram of the image reconstruction of ${ }^{88} \mathrm{Y}$ source. The counts are normalized by the area.
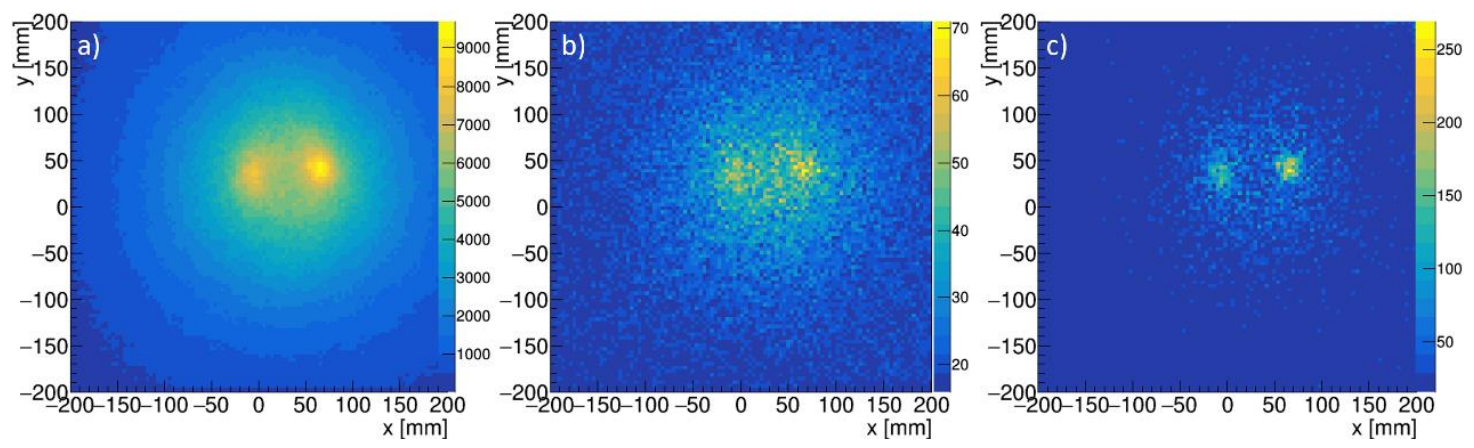

Figure 9. Image reconstruction from two sources, ${ }^{57} \mathrm{Co}$ (left) and ${ }^{139} \mathrm{Ce}$ (right). a) is simple back projection using SOE initialisation (multiple points per event), b) is the image from SOE initilisation and c) is the image after applying 50 iterations. 
Another image reconstruction example is shown in Figure 9b and Figure 9c, where the image of two sources is reconstructed before SOE and after 50 iterations, respectively. After the iterations, the sources are better resolved and the background around the sources decreases. The image reconstruction using SOE can potentially be further improved by implementing resolution recovery [17].

\section{Conclusion}

We have shown the status of a semiconductor Compton camera for prompt gamma imaging during proton beam therapy. SOE image reconstruction has been used and it was demonstrated that image resolution improvements can be made reasonably quickly. Further speed improvements can be gained by implementing multicore and GPU acceleration. The next steps include testing the Compton camera at the Clatterbridge Cancer Centre, where the gamma background have already been assessed.

\section{Acknowledgments}

We are grateful to the staff at the Clatterbridge Cancer Centre NHS Foundation Trust, without who the proton beam measurements could not have taken place. This research was supported by STFC CLASP grant (ST/M007898/1).

\section{References}

[1] H. Paganetti, Range uncertainties in proton therapy and the role of Monte Carlo simulations, Phys. Med. Biol. 57 (2012) R99-R117.

[2] A.-C. Knopf and A. Lomax, In vivo proton range verification: a review, Phys. Med. Biol. 58 (2013) R131-R160.

[3] C. H. Min et al., Prompt gamma measurements for locating the dose falloff region in the proton therapy, Appl. Phys. Lett. 89 (2006) 183517.

[4] C. Richter et al., First clinical application of a prompt gamma based in vivo proton range verification system, Radiother Oncol. 118 (2016) 232-237.

[5] C. H. Min et al., Development of array-type prompt gamma measurements system for in vivo range verification in proton therapy, Med Phys. 39 (2012) 2100-2107.

[6] F. Roellinghoff et al., Design of a Compton camera for 3D prompt gamma imaging during ion beam therapy, Nucl Instr Meth Phys Res A 648 (2011) S20-S23.

[7] S. W. Peterson et al., Optimizing a 3-stage Compton camera for measuring prompt gamma rays emitted during proton radiotherapy, Phys. Med. Biol. 55 (2010) 6841-6856 (2010).

[8] S. Agostinelli et al., Geant4 - a simulation toolkit, Nucl Instr Meth Phys Res A 506 (2003) 250-303.

[9] A. Kacperek, Protontherapy of eye tumours in the UK: A review of treatment at Clatterbridge, Appl. Radiat. Isot. 67 (2009) 378-386.

[10] J. M. Verburg et al., Simulation of prompt gamma-ray emission during proton radiotherapy, Phys. Med. Biol. 57 (2012) 5459-5472.

[11] A. Kacperek, Dose verification by activation in vivo following proton beam eye radiotherapy, $J$. Radioanal. Nucl. Chem. 271 (2007) 731-740.

[12] J. Jeyasugiththan and S. W. Peterson, Evaluation of proton inelastic reaction models in Geant4 for prompt gamma production during proton therapy, Phys. Med. Biol. 60 (2015) 19.

[13] M. Pinto et al., Assessment of Geant4 prompt gamma emission yields in the context of proton therapy monitoring, Front Oncol 6 (2016) 10. 
[14] B. Smith, Reconstruction methods and completeness conditions for two Compton data models, J. Opt.Soc. Am. A 22 (2005) 445-459.

[15] A. Andreyev et al., Stochastic image reconstruction method for Compton camera, in Proc. IEEE Nucl. Sci. Symp. Conf. Record (NSS/MIC), Orlando, FL U.S.A October 24 November 1 2009, pg. 2985-2988.

[16] L.J. Harkness et al. Characterisation of a Si (Li) orthogonal-strip detector, Nucl Instr Meth Phys Res A 726 (2013) 52-59.

[17] A. Andreyev et al., Resolution recovery for Compton camera using origin ensemble algorithm, Med. Phys. 43 (2016) 4866-4876. 